\title{
Design and Implementation of Cherry Picking Robot Vision Recognition System Based on Face Recognition
}

\author{
Qi Rong ZHANG ${ }^{a}$, Ru Hui HU and Ting Zhong YU \\ Information Engineering, Guizhou University of Engineering Science, Bijie 551700, Guizhou, China
}

\begin{abstract}
This paper analyzes the necessity of cherry picking robot vision recognition system. It introduces the system function and the system structure, and provides the system operation environment, the development platform and the database design. This paper designs and implements the cherry picking robot vision recognition system, based on C\# technology, using MATLAB, using face recognition technology.
\end{abstract}

Keywords. face recognition; Cherry picking robot; Vision recognition system

\section{Introduction}

Along with the development of agriculture in our country, in order to reduce the cost and improve the productivity, the requirement of the agricultural mechanization is more and more high. Cherry's price is higher, all over the country in the development of cherry cultivation. Cherry planting difficulty is not great, but its picking cost of artificial is more, which is a reason for its high cost, so it is imperative to use the robot picking cherries. But if the price of cherry robot is high, it is bound to cause its promotion difficulties and the value of the use of reduced. This system uses C\# and MATLAB mixed programming technology, which can realize the basic function of cherry picking robot vision recognition system. In addition, the price of computer hardware is reduced, which makes it possible to extend the computational intelligence to the practical robot. So this system will use face recognition technology to replace the general system using password function, bring convenience to users. It prevent forget passwords and password to remember, at the same time, to prevent theft cherry picking robot. No face recognition successful login, cherry picking robots will not be able to work.

\section{Face recognition technology}

Face recognition is new technology in the development of recent decades, which has great practical value. Face recognition is based on multiple disciplines on the basis of a comprehensive technology. With the development of The Times, people is higher and higher requirements for protection of privacy, therefore, it produced a lot of way

\footnotetext{
${ }^{\mathrm{a}}$ Qi Rong ZHANG: zqr_hk@163.com
}

to protect privacy, such as using the id, password and smart card, etc. But these methods are not convenient, easy loss, easy to forget, theft, which can not adapt to the need for the development of the society. So the researchers began to target the biological recognition technology. This technology not only has the uniqueness of the individual, but these features are usually more stable. The biometric technologies mainly include: face recognition, iris recognition, fingerprint recognition, voice recognition, etc. Compared with other biometric, facial recognition has the friendly, convenient and not easy to forge, natural advantages, at the same time face data collection is also relatively simple. The cost is not high, not psychological barriers for being collected. Face recognition system is not high cost, more and more people to study it [1-4]. It has been widely used in many fields, such as the entrance of Beijing Olympic Games, such as in Xinjiang highway, of all the passengers were identity cards and the actual face matching for face recognition, to find wanted terrorist criminals, such as the Hong Kong customs using face recognition technology for identification.

\section{System design}

\subsection{System architecture}

The cherry picking robot visual recognition system based on face recognition has four function modules. They are face recognition login module, image acquisition module, cherry recognition module, binocular positioning module. 
The overall structure of the system is shown in the following figure.

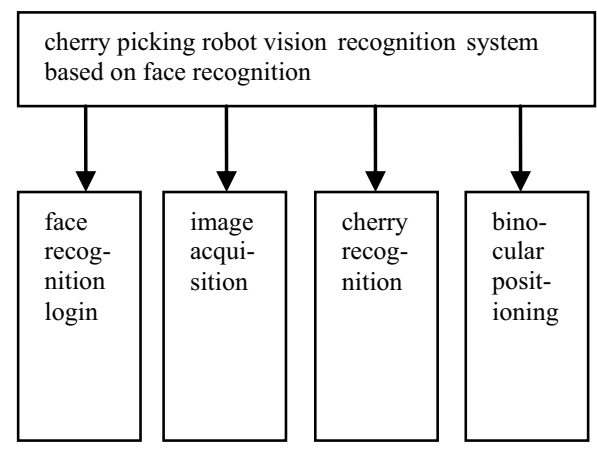

Figure 1. The overall structure of the system

As follows, we illustrate the functions of each subsystem.

Users can through the face recognition login module, after the camera to take pictures to identify the successful to login system. The module includes access to the image, image preprocessing, face detection, face recognition, face comparison of 5 sub functions. User face database is established in the function, because the users of the system is not much, and shooting is according to the positive, so recognition speed is fast and recognize faces time is short, accord with the actual needs. But the difficulty is only a test image, large difficulties of identification. Then we enter the image acquisition module, the system through the module collected in the actual picking environment of the cherry image, and then the image is stored in the module. Then the most important module of the system is cherry recognition module. In this module, eight sub function operations are performed on the cherry image, which are median filtering, color recognition, fixed threshold segmentation, morphological opening operation, morphological closing operation, Canny operator and circle Hough transform, picking point acquisition. Through a series of operations of the module, the cherry of the cherry image was identified by the picking point, so the cherry picking robot can pick cherry by the robot hand. The function of the module is sequential, one by one to carry out the operation, which can not be any choice of a sub module to perform. You must complete all the sub function, cherry recognition function to complete. This is different from general software system, which can choose subsystem operation according to the user. Binocular positioning module is through the left and right camera to get the internal calibration parameters on both sides, and then gets the external calibration parameters. The 3D coordinates of picking point can be obtained by the internal and external calibration parameters.

\subsection{Development environment}

Cherry picking robot vision recognition system based on face recognition selects $\mathrm{C} \#$ as the system development language and Microsoft Visual Studio 2010 for online system environment, using MATLAB image processing, with SQL Server 2010 as the system database on the Server. The development process and the debugging of the whole system are conducted on Microsoft's Windows 7 operating system. Cherry picking robot visual recognition system based on face recognition uses SQL Server 2010 to build the database server. The database involves the main data table including the users table, cherry collection images table, cherry circle Hough transform table, cherry picking point table and cherry three dimensional picking point table. Class diagram of data relationship between them is shown in table 1 .

Table 1. List of main data tables

\begin{tabular}{|c|c|c|}
\hline $\begin{array}{c}\text { The } \\
\text { serial } \\
\text { number }\end{array}$ & The name of the table & explain \\
\hline 1 & Users & users table \\
\hline 2 & CollectionImages & $\begin{array}{c}\text { cherry } \\
\text { collection } \\
\text { images } \\
\text { table }\end{array}$ \\
\hline 3 & CircleHoughTransform & $\begin{array}{c}\text { cherry } \\
\text { circle } \\
\text { Hough } \\
\text { transform } \\
\text { table }\end{array}$ \\
\hline 4 & PickingPoint & $\begin{array}{c}\text { cherry } \\
\text { picking } \\
\text { point table }\end{array}$ \\
\hline 5 & ThreeDimensionalPickingPoint & $\begin{array}{c}\text { cherry three } \\
\text { dimensional } \\
\text { picking } \\
\text { point table. }\end{array}$ \\
\hline
\end{tabular}

The following is a list of the main data tables.

(1) The user table: this table is used to store the information of the user.

(2) Cherry collection images: the table used to store the actual scene of the camera to capture the image of the cherry.

(3) Cherry circle Hough transform table: the table is used to store the image cherry the circle Hough transform of image recognition module.

(4) Cherry picking point table: this table is used to store the image after the circle Hough transform.

(5) Cherry three dimensional picking point table: this table is used to store the cherry image, which is determined by the three-dimensional binocular calibration picking point.

\subsection{The key technology of system implementation}

The database stores a large amount of data, so design of the database directly affects the realization of the system. The database of the cherry picking robot vision recognition system is a very important part of the system, and a large number of data are stored in the database. Cherry picking robot vision recognition system includes a number of functional modules, so the following is part of the code.

(1) Part of the database connection code is as follows: [5] //The database connection 


\section{string}

dat="server=.;database="+textBox1.Text.Trim ()$+" ; u$

$\mathrm{id}=$ sa;pwd=";

connn=new $\quad$ SqlConnection(dat);//Create a

SqlConnection object

connn.Open(); // Open a connection

if $($ connn,State $==$ ConnectionState.Open $) / /$ Determine the current connection status

\{

MessageBox.Show( "Connect successfully” ); //

Pop-up prompts

//Close the connection

String dat = " ";// Define a string variable

Connn.Close();//Use Close to close the connection

If(connn. State $==$ ConnetionState.Closed) // To

determine whether the current connection closed

\{

dat= "Database has been successfully closed $\backslash n$ ” ; //

If it has been closed, then pop-up tips

\}

(2) Face recognition part of the MATLAB code t_all=zeros(nr*nc,tp*ts); \% The initial training sample set is 0

for num_tp $=1: t p$

res $=[]$;

for num ts $=1$ :ts

num_sequ $=$ sequ(num_ts);

img_name $=$ strcat $(' \mathrm{C}: \mid$ Documents and

Settings $\backslash$ Administrator

$\backslash$ ModifyFERET $\backslash$ ModifyFERET $\backslash$ ','s',int2str(num_tp), '’',int

2str(num_sequ),'.jpg');

img=imread(img_name); $\%$ Read the face image img $=$ double(img)./256;

img=imresize(img, [12 12]); \%Cut out the image of $12 \times 12$

mid $=($ num_tp-1)*ts + num_ts;

res=reshape (img,size (img, 1$) * \operatorname{size}(i m g, 2), 1)$;

t_all(:,mid $)=$ res; \%Read the images are put into the training sample set

end

end

(3) Median filter MATLAB code

Img=imread('img.tif');

Noi=imnoise(Img,'salt \& pepper',0.02); \% Add salt, pepper, noise density of 0.02

$\mathrm{KK} 1=$ medfilt2(Noi); $\quad \%$ Median filtering is performed in a $3 \times 3$ neighborhood window.

KK2 $=$ medfilt2(Noi,[5 5]); \% Median filtering is performed in $5 * 5$ neighborhood windows

\section{Conclusion}

The system can complete the visual identification of the cherry in the cherry orchard, so cherry picking robot can run smoothly. With the maturity of face recognition technology, the continuous improvement of computer hardware performance and the continuous decline in hardware prices, it is possible to use face recognition technology in the cherry picking robot vision recognition system. Cherry recognition module is the most critical modules in this system. This system can also apply to other fruit and vegetable picking robot visual recognition, which is for fruits and vegetables with a large difference in color and background color, and shape close to the circular shape.

\section{Acknowledgments}

This work was supported by 2015 Annual Science and Technology Department of Guizhou Province, Bijie City Science and Technology Bureau, Guizhou University of Engineering Science Joint Science and Technology Fund Project, Cherry picking robot vision system research, Guizhou Branch of LH word [2015] No. 7599; the grants from 2013 Annual Science and Technology Department of Guizhou Province, Bijie City Science and Technology Bureau, Bijie University Joint Fund Scheme under Project No. Guizhou Branch of J word LKB [2013] No.14.

\section{References}

1. S. Dongmei, Q. Zhengding. Review of biometric identification technology [J]. Electronic journal, 2001,12A (12) : 1744-1748

2. T. Jie, Y. Xing. Theory and application of biometric identification technology [M]. Beijing: electronic industry press, 2005

3. Qi Rong Zhang, Zhongshi HE, The Twodimensional Bidirectional Inverse Laplacian Margin Maximum Criterion for Face Recognition, Journal of Computational Information Systems, 2010, 6: 11011109.

4. Qi Rong Zhang, Zhongshi HE, Dual-tree Quaternion Wavelets for Face Recognition, Journal of Computational Information Systems, 2010, 6: 11511160

5. Tomorrow Technology. C \# from entry to the master (3rd edition) [M]. Beijing: Tsinghua University press.2012. 9 . 